\title{
ATENUACIÓN DE CAMPOS ELECTROMAGNÉTICOS EN INSTRUMENTOS AERONÁUTICOS EMPLEANDO MANUFACTURA ADITIVA
}

\author{
Lasluisa Héctor ${ }^{1}$, Capelo Aldo ${ }^{2}$ \\ hector_hgln@hotmail.com ${ }^{1}$, aldogrivaldy2005@hotmail.com² \\ https://orcid.org/0000-0001-7355-913711, https://orcid.org/0000-0003-2409-0875² \\ Universidad de las Fuerzas Armadas-ESPE \\ Quito-Ecuador
}

Recibido (05/08/20), Aceptado (17/08/20)

\begin{abstract}
Resumen: Esta investigación explora la utilización de materiales alternativos como el filamento termoplástico ABS y un moderno método de manufactura, como lo es la impresión 3D para reproducir la estructura de un instrumento medidor de revoluciones por minuto RPM de un motor aeronáutico con características de atenuación de campos electromagnéticos y supresión de ruido, similares a los instrumentos originales producidos con aluminio embutido, para lograr este propósito se emplea la ley de Gauss y el efecto de la jaula de Faraday, además mediante la combinación de plástico y fibra de vidrio se logra un sistema de paredes dobles que atenúan la propagación del sonido. Empleando el diseño e ingeniería asistidos por computador se ejecuta la simulación y validación del prototipo empleando el método de análisis por elementos finitos y análisis de compatibilidad electromagnética, finalmente los resultados de las pruebas de laboratorio y de campo permiten cuantificar las nuevas características mecánicas obtenidas.
\end{abstract}

Palabras Clave: Impresión 3D, instrumento aeronáutico, jaula de Faraday.

\section{ATTENUATION OF ELECTROMAGNETIC FIELD IN FLIGHT INSTRUMENT USING ADDITIVE MANUFACTURING}

\begin{abstract}
This research explores the use of alternative materials like this ABS thermoplastic filament and a modern manufacturing method, such as 3D printing to reproach the enclosure of a tachometer indicating instrument that measures revolutions per minute RPM of an aeronautical gas turbine engines with characteristics of attenuation of electromagnetic fields and noise suppression, same to the original instrument enclosure made by extruded aluminum, to find this goal uses Gauss's law and the Faraday cage, also, the combination of plastic and fiberglass like a double walls structure that attenuates noise propagation. Use computer-aided engineering and design, by the simulation and validation of the prototype characteristics through the method of finite element and electromagnetic compatibility analysis. Finally, the results of laboratory and field tests allow quantifying the new mechanical characteristics acquired.
\end{abstract}

Keywords: 3D Print, flight instrument, Faraday cage. 


\section{I.INTRODUCCIÓN}

Los sistemas de aviónica representan el 30\% del costo total de una aeronave y su tecnología está en constante evolución [1], lo que ha generado que ciertas aeronaves de uso militar en el Ecuador con un promedio de 30 años de antigüedad, vean reducida la disponibilidad de los repuestos de estos sistemas.

Los componentes estructurales de los instrumentos aeronáuticos de vuelo están fabricados en aluminio mediante embutido en frío, este método es empleado para la producción en masa [2], por lo que resulta una excesiva inversión para la manufactura de pocos objetos.

Sin embargo, en los últimos años la manufactura aditiva se ha venido abriendo paso para ofrecer soluciones prácticas en la creación de objetos de múltiples formas, y en la actualidad ya es empleada en varios campos de la industria [3].

En este trabajo se propone emplear la manufactura aditiva para reproducir los componentes estructurales de los instrumentos de medición de revoluciones por minuto de los sistemas de propulsión aeronáuticos, con características de atenuación de campos electromagnéticos y de ruido, similar en forma y funcionalidad a los componentes originales.

El problema de estudio consiste en lograr que el material plástico ABS empleado en la impresión 3D asemeje las características mecánicas del aluminio embutido, como la resistencia mecánica a esfuerzos, atenuación de campos electromagnéticos y ruido. Para cumplir con este propósito se empleará una combinación con materiales como una malla metálica y una capa de fibra de vidrio.

Dentro de la industria aeronáutica, la impresión 3D ya es utilizada para crear piezas funcionales de aviones comerciales, debido a la facilidad que presta para manufacturar piezas complejas, como lo señala Peter Sander Gerente de innovación de conceptos tecnológicos de la Compañía Airbus S.A.S [4], evidenciando así la factibilidad del presente trabajo.

Para la atenuación de los campos electromagnéticos, es posible emplear el efecto de la Jaula de Faraday que consiste en lograr que el campo electromagnético dentro de un conductor sólido o hueco sea nulo, ante la presencia de campos de excitación externa. La jaula de Faraday y el campo nulo dentro de la misma se puede verificar mediante la ley de Gauss para una superficie cerrada [5]. Se realizará el modelaje y diseño de una estructura metálica tipo malla para la atenuación de campos electromagnéticos de hasta 100 micro Teslas y 136,99 Mhz de frecuencia, que será integrada a la estructura de plástico ABS.

La atenuación de ruido se logra aplicando el principio de las paredes dobles, el cual es un método sencillo y económico para aumentar el aislamiento acústico de una pared simple, evitando así la propagación de ondas sonoras en un espacio específico [6]. Bajo este principio se realiza el diseño y construcción de una estructura de plástico ABS de pared doble, y en medio de estas paredes se incluye la fibra de vidrio, que es un material de diferente densidad y porosidad que logra el efecto de reducir la propagación de las ondas sonoras.

Así, empleando el diseño e ingeniería asistidos por computador se realiza la validación con el método de elementos finitos (CAE) y el método de análisis electromagnético (EMAG) del nuevo prototipo, y además los ensayos y pruebas de campo permiten la comprobación de los resultados.

En la sección II se presenta el fundamento teórico de la jaula de Faraday, y la propagación de ruido en estructuras de paredes dobles.

La sección III detalla los procesos consecutivos desarrollados durante este trabajo, se inicia implementando los modelos matemáticos de Gauss para nuestro caso, continua con el diseño asistido por computador, validación mediante simulación por software, luego se realiza la manufactura del prototipo y finaliza con las respectivas pruebas de campo.

Los resultados de la presente investigación son presentados en la sección IV.

\section{II.DESARROLLO}

\section{A.Interferencias en dispositivos de medición.}

Para dispositivos electrónicos las interferencias originadas por las ondas electromagnéticas y las ondas sonoras son las que incidirán en el buen funcionamiento de los mismos [7].

\section{B.Ondas electromagnéticas}

Son fenómenos de propagación de energía sin transporte de materia, la particularidad de estas ondas reside en que no necesitan un medio material para su propagación. La radiación electromagnética posee dos campos perpendiculares oscilantes entre sí, uno es el campo eléctrico y otro es el campo magnético [5]. 


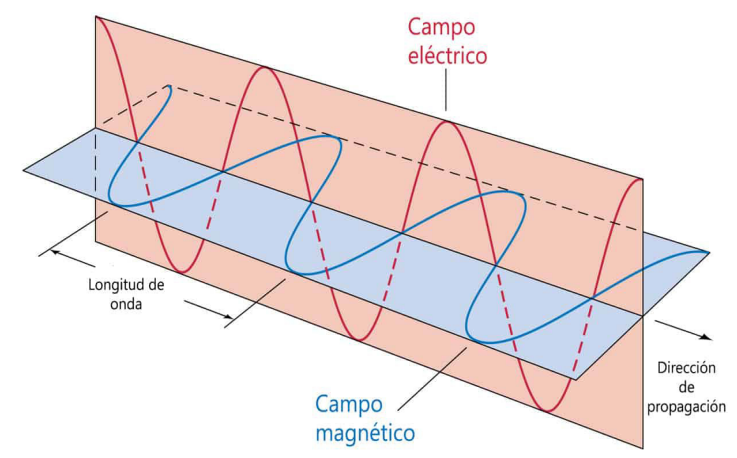

Fig. 1. Representación de una onda electromagnética

\section{C.Atenuación de ondas electromagnéticas.}

La electrostática y electromagnetismo nos proporcionan las bases para evitar la interferencia que producen las ondas electromagnéticas, en específico la ley de Gauss y una aplicación de esta ley denominada jaula de Faraday [8].

\section{D.Jaula de Faraday.}

La Jaula de Faraday es el efecto en el cual el campo electromagnético dentro de un conductor sólido o hueco es nulo ante la presencia de campos de excitación externa, este efecto consiste en que el conductor se polariza con una carga negativa a un lado y con carga positiva en el lado opuesto anulando así el campo interno, esto se puede verificar mediante la ley de Gauss [5].

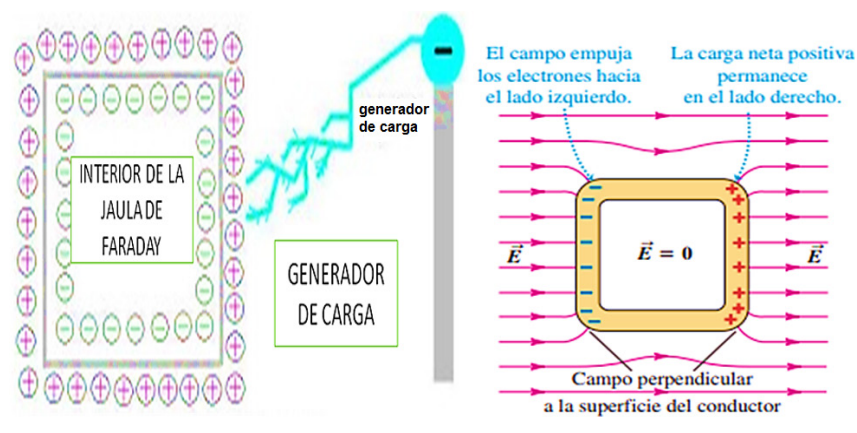

Fig. 2. Representación del fenómeno de aislamiento de la jaula de Faraday

\section{E.Ondas sonoras.}

Las ondas sonoras son ondas mecánicas longitudinales, se consideran mecánicas porque necesitan un medio material para su propagación, y longitudinales porque las partículas del medio actúan en la misma dirección en la que se propaga la onda [6].

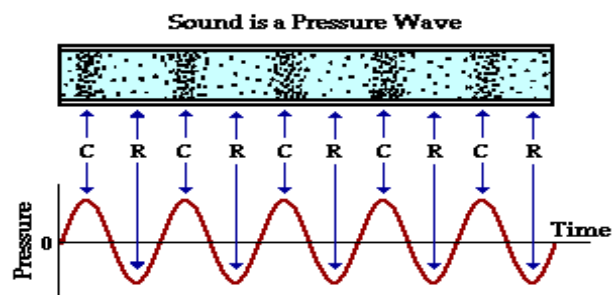

\section{F.Paredes dobles.}

Este método consiste en formar una estructura de dos paredes, y que en el medio de estas se forme una cavidad de separación, dicha cavidad deberá ser pequeña en relación a la longitud de onda del sonido y deberá albergar un material con características de absorbente acústico [6].

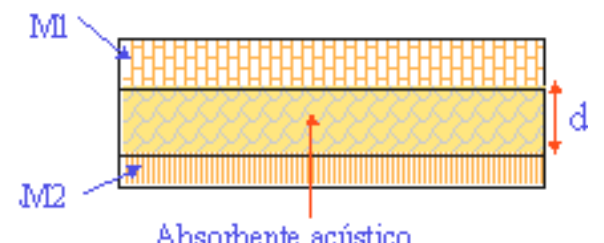

Fig. 4. Pared doble con material absorbente en la cavidad

\section{III.METODOLOGÍA}

A.Diseño de la jaula de Faraday.

Según la ley de Gauss una carga externa proyecta un cono con un ángulo sólido $\mathrm{d} \Omega$ he intercepta la superficie $\mathrm{S}$ en $\mathrm{dS}_{1} \mathrm{y} \mathrm{dS}_{2}[9]$.

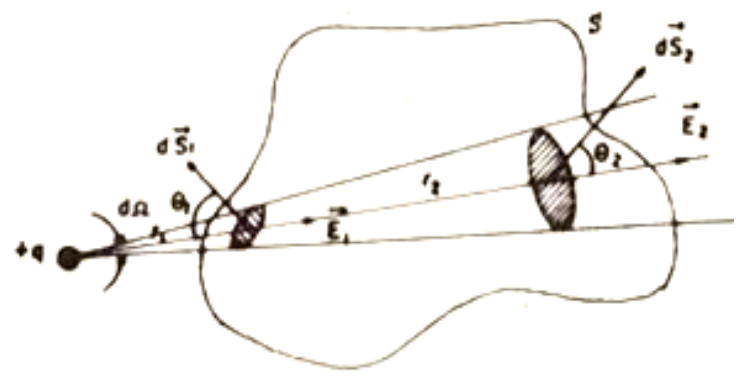

Fig. 5. Superficie cerrada (Jaula de Faraday), excitada por una carga externa

También nos indica que el flujo eléctrico es directamente proporcional, al campo eléctrico y a la superficie.

$$
\phi=\oint \vec{E} \cdot d \vec{s}=\frac{q}{\epsilon_{0}}
$$


Donde:

$\phi$ : flujo eléctrico.

$\overrightarrow{\mathrm{E}}$ : campo eléctrico.

$\mathrm{d} \overrightarrow{\mathrm{s}}$ : diferencial de superficie.

q: carga eléctrica.

\section{B.Selección del material para la Jaula de Faraday}

El primer requisito es que el material sea de tipo conductor, ya que la atenuación del campo es proporcional a la propiedad de conductividad eléctrica.

$$
\epsilon_{0}=8.854 * 10^{-12}\left[\frac{\mathrm{C}^{2}}{\mathrm{Nm}^{2}}\right] \quad \text { : permeabilidad del vacío. }
$$

TABLA I. Conductividad para materiales

\begin{tabular}{cccc}
\hline Tipo & Material & Conductividad eléctrica $\left(\mathrm{Sm}^{-1}\right)$ & Temperatura $\left({ }^{\circ} \mathrm{C}\right)$ \\
\hline & Grafeno & $9.87^{*} 10^{7}$ & 20 \\
Clata & $6.30^{*} 10^{7}$ & 20 \\
& Cobre & $5.96^{*} 10^{7}$ & 20 \\
& Oro & $4.55^{*} 10^{7}$ & $20-15$ \\
& Aluminio & $3.78^{*} 10^{7}$ & 20 \\
& Hierro & $1.53^{*} 10^{7}$ & 20 \\
\hline
\end{tabular}

$$
\delta=\frac{1}{\sqrt{\pi f \mu_{0} \mu_{r} \sigma}}
$$

Donde:

$\delta$ : profundidad de penetración.

f: frecuencia.

$\mu_{0}=\mu_{0}=4 \pi * 10^{-7}\left[\frac{\mathrm{Vs}}{\mathrm{Am}}\right]$ : permeabilidad del vacío.

$\mu_{\mathrm{r}}$ : permeabilidad relativa del material.

$\sigma$ : conductividad eléctrica.

La frecuencia más alta empleada en aviación es de 136.99[MHz], y para el caso del acero de bajo carbono tenemos:

$$
\begin{gathered}
\delta=\frac{1}{\sqrt{\pi * 136.99 * 10^{6}[\mathrm{~Hz}] * 4 \pi * 10^{-7}\left[\frac{\mathrm{VS}}{A m}\right] * 2420 * 0.704 * 10^{7}\left[\frac{1}{\Omega \mathrm{m}}\right]}} \\
\delta=3.294 * 10^{-7}[\mathrm{~m}] \\
\delta=3.294 * 10^{-4}[\mathrm{~mm}]=0.0003294[\mathrm{~mm}]
\end{gathered}
$$

Como el espesor de la jaula debe ser mayor a la profundidad de penetración, y ya que el valor calculado es pequeño, se aplica un factor de seguridad alto acorde a un espesor de malla comercial.

$$
e_{\text {jaula }}=F S * \delta
$$

Tomando un factor de FS=800, tenemos:

$$
\begin{gathered}
e_{\text {jaula }}=800 * 0.0003294[\mathrm{~mm}] \\
e_{\text {jaula }}=0.264[\mathrm{~mm}] \approx 0.28[\mathrm{~mm}]
\end{gathered}
$$

El diámetro del alambre que estará constituida la malla debe ser de $0.28[\mathrm{~mm}]$.

\section{D.Tamaño de rejillas para la Jaula de Faraday}

Para evitar que las ondas electromagnéticas atraviesen los agujeros de la jaula de Faraday, la malla debe tener orificios más pequeños que la longitud de onda de los campos externos.

$$
f=\frac{c}{\lambda}
$$

Donde

f: frecuencia de las ondas electromagnéticas.

$\lambda$ : tamaño de longitud de onda.

$c=3 * 10^{8}\left[\frac{m}{s}\right]:$ velocidad de la luz en el vacío.

Entonces:

$$
\begin{gathered}
\lambda=\frac{c}{f} \\
\lambda=\frac{3 * 10^{8}\left[\frac{\mathrm{m}}{\mathrm{s}}\right]}{136.99 * 10^{6}[\mathrm{~Hz}]}
\end{gathered}
$$

$\lambda=2.19 \mathrm{~m}=2190[\mathrm{~mm}]$ longitud máxima de las rejillas Debido al valor alto de longitud de onda podemos ocupar un factor de seguridad alto para obtener una medida comercial.

$$
\begin{gathered}
F S=2000 \\
w=\frac{\lambda}{F S} \\
w=\frac{2190[\mathrm{~mm}]}{2000}
\end{gathered}
$$


$w=1.1[\mathrm{~mm}] \approx 1.26[\mathrm{~mm}]$ Longitud de la luz de la malla

\section{E.Selección de la malla metálica en catálogo}

TABLA II. Material para la malla

\begin{tabular}{ccc}
\hline \multicolumn{3}{c}{ Malla Galvanizada } \\
\hline Código & Luz & Alambre \\
L1815060 & $1,26 \mathrm{~mm}$ & $0,28 \mathrm{~mm}$ \\
\hline
\end{tabular}

\section{F.Diseño del aislamiento acústico de pared doble}

$\mathrm{La}$ frecuencia que alcanza el ruido externo es de $\mathrm{f}=15000 \mathrm{~Hz}$. Primero se calcula la frecuencia de resonancia del aislamiento acústico utilizando la fórmula general para aislamientos de paredes compuestas:

$$
f_{0}=\frac{1}{2 \pi} \sqrt{k \frac{M 1+M 2}{M 1 * M 2}}
$$

Donde:

$\mathrm{f}_{-0}$ : Frecuencia de resonancia.

: Peso específico del material absorbente.

M1: densidad superficial de la pared $1 .\left[\mathrm{kg} / \mathrm{m}^{2}\right]$

M2: densidad superficial de la pared 2. $\left[\mathrm{kg} / \mathrm{m}^{2}\right]$

Se procede a calcular las densidades superficiales de las dos paredes (M1 y M2).

$$
\begin{aligned}
& M 1=\frac{m}{A}=\frac{\rho V}{A} \\
& M 1_{A B S}=\frac{\rho_{A B S} * V}{A} \\
& M 1_{A B S}=\frac{\rho_{A B S} * \pi * h\left(r_{1}{ }^{2}-r_{2}{ }^{2}\right)}{2 \pi r_{1} h}=\frac{\rho_{A B S}\left(r_{1}{ }^{2}-r_{2}{ }^{2}\right)}{2 * r_{1}} \\
& M 1_{A B S}=\frac{\rho_{A B S}\left(r_{1}{ }^{2}-r_{2}{ }^{2}\right)}{2 * r_{1}} \quad M 2_{A B S}=\frac{\rho_{A B S}\left(r_{3}{ }^{2}-r_{4}{ }^{2}\right)}{2 * r_{3}}
\end{aligned}
$$

En base a la disposición de las dimensiones de las dos paredes, así como de la cavidad mostradas en la figura 6 los radios tomarían los siguientes valores:

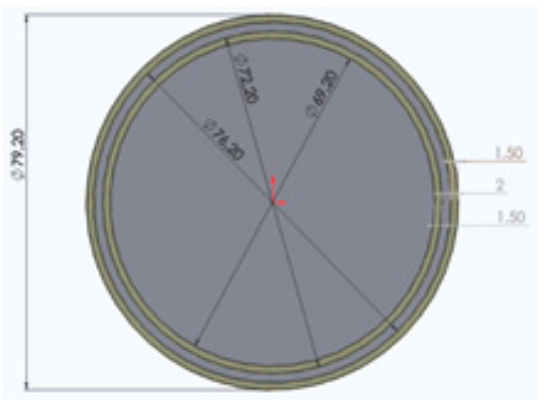

Fig. 6. Disposición de diámetros del cilindro

$$
\begin{aligned}
& r_{1}=39.6 \mathrm{~mm}=0.0396 \mathrm{~m} \\
& r_{2}=38.1 \mathrm{~mm}=0.0381 \mathrm{~m} \\
& r_{3}=36.6 \mathrm{~mm}=0.0366 \mathrm{~m} \\
& r_{4}=35.1 \mathrm{~mm}=0.0351 \mathrm{~m} \\
& \rho_{A B S}=1.05 \frac{\mathrm{g}}{\mathrm{cm}^{3}}=1050 \frac{\mathrm{kg}}{\mathrm{m}^{3}}
\end{aligned}
$$

Reemplazando los valores de los radios en las ecuaciones de M1 Y M2 obtenemos:

$$
\begin{aligned}
& M 1_{A B S}=\frac{\rho_{A B S}\left(r_{1}{ }^{2}-r_{2}{ }^{2}\right)}{2 * r_{1}}=\frac{1050 \frac{\mathrm{kg}}{\mathrm{m}^{3}}\left(0.0396^{2}-0.0381^{2}\right) \mathrm{m}^{2}}{2(0.0396 \mathrm{~m})}=1.5452 \frac{\mathrm{kg}}{\mathrm{m}^{2}} \\
& M 2_{A B S}=\frac{\rho_{A B S}\left(r_{3}{ }^{2}-r_{4}{ }^{2}\right)}{2 * r_{3}}=\frac{1050 \frac{\mathrm{kg}}{\mathrm{m}^{3}}\left(0.0366^{2}-0.0351^{2}\right) \mathrm{m}^{2}}{2(0.0366 \mathrm{~m})}=1.5427 \frac{\mathrm{kg}}{\mathrm{m}^{2}}
\end{aligned}
$$

Se calcula el valor del peso específico del material que se encuentra entre las dos paredes en este caso fibra de vidrio.

$$
\begin{gathered}
\rho_{\text {fibra de vidrio }}=2.35 \frac{\mathrm{g}}{\mathrm{cm}^{3}}=2350 \frac{\mathrm{kg}}{\mathrm{m}^{3}} \\
k=\rho_{\text {fibra de vidrio }} * g \\
k=2350 \frac{\mathrm{kg}}{\mathrm{m}^{3}} * 9.81 \frac{\mathrm{m}}{\mathrm{s}^{2}}
\end{gathered}
$$




$$
k=23053.5 \frac{\mathrm{kg}}{\mathrm{m}^{2} \mathrm{~s}^{2}}=23053.5 \frac{\mathrm{N}}{\mathrm{m}^{3}}
$$

Reemplazando todos los datos obtenidos para calcular la frecuencia de resonancia.

$$
\begin{gathered}
f_{0}=\frac{1}{2 \pi} \sqrt{k \frac{M 1+M 2}{M 1 * M 2}} \\
f_{0}=\frac{1}{2 \pi} \sqrt{23053.5 \frac{\mathrm{N}}{\mathrm{m}^{3}} * \frac{1.5452 \frac{\mathrm{kg}}{\mathrm{m}^{2}}+1.5427 \frac{\mathrm{kg}}{\mathrm{m}^{2}}}{1.5452 \frac{\mathrm{kg}}{\mathrm{m}^{2}} * 1.5427 \frac{\mathrm{kg}}{\mathrm{m}^{2}}}} \\
f_{0}=\frac{1}{2 \pi} \sqrt{23053.5 \frac{\mathrm{N}}{\mathrm{m}^{3}}\left(1.29538 \frac{\mathrm{m}^{2}}{\mathrm{~kg}}\right)} \\
f_{0}=27.51 \mathrm{~Hz}
\end{gathered}
$$

Existen tres casos en relación a la frecuencia de resonancia y la frecuencia con la que se realiza el análisis, con respecto a paredes compuestas para el aislamiento acústico [6].

La pared extra no es efectiva bajo la frecuencia de resonancia, $f<f_{0} \operatorname{con} R_{E} \approx 0$

En la frecuencia de resonancia $\mathrm{f}=\mathrm{f}_{0}$ empeora el aislamiento.

A partir de la frecuencia de resonancia $\mathrm{f}>\mathrm{f}_{0}$ la pared extra aporta un mejoramiento aproximado $\mathrm{R}_{\mathrm{E}} \approx 40 \log (\mathrm{f} /$ $\mathrm{f}_{0)}$. En conclusión, como $\mathrm{f}>\mathrm{f}_{0}$, existirá aislamiento acústico.

\section{Distancia entre las paredes de aislamiento}

La segunda pared debe instalarse con una cierta separación de la pared simple, se puede asumir que el espacio entre las paredes, cuyo espesor es pequeño en relación a la longitud de onda, actuará como un resorte con rigidez por unidad de superficie.

$$
\begin{aligned}
& \mathrm{v}=343 \frac{\mathrm{m}}{\mathrm{s}} \mathrm{v} \text { : velocidad de propagación del sonido en el aire } \\
& \lambda=\mathrm{v} * \mathrm{~T}=\frac{\mathrm{v}}{\mathrm{f}}
\end{aligned}
$$

Donde:

$\lambda$ : longitud de onda, f: frecuencia

$$
\lambda=\frac{\mathrm{v}}{\mathrm{f}}=\frac{343 \mathrm{~m} / \mathrm{s}}{15000 \mathrm{~Hz}}=22.867[\mathrm{~mm}] \text { Máxima separación entre las paredes. }
$$

De acuerdo al diseño se asume una distancia de $2 \mathrm{~mm}$ de separación entre las paredes.

\section{H.Selección del material absorbente acústico}

Para evitar posibles resonancias en la cavidad que se genera por la separación entre las dos paredes se debe introducir en dicho espacio un material absorbente poroso [6].

La fibra de vidrio no retiene el agua, posee una es-

\begin{tabular}{|c|c|c|c|}
\hline Densidad & $1,8 \mathrm{~kg} / \mathrm{dm} 3$ & Dureza Barcol & $35 / 45$ \\
\hline Resistencia Axial & $4,9 \mathrm{~kg} / \mathrm{mm} 2$ & $\begin{array}{l}\text { Coef. Conductividad } \\
\text { térmica }\end{array}$ & $\begin{array}{c}0,15-0,20 \\
\mathrm{kcal} / \mathrm{hm}^{\circ} \mathrm{C}\end{array}$ \\
\hline $\begin{array}{l}\text { A tracción Circunf. } \\
\text { A comprensión }\end{array}$ & $17,0 \mathrm{~kg} / \mathrm{mm} 2$ & Coef. Perdida de carga CW & 150 \\
\hline Circunf. & $6,9 \mathrm{~kg} / \mathrm{mm} 2$ & Resistencia eléctrica. & $1011 \mathrm{ohm} / \mathrm{cm}$ \\
\hline A flexión Circunf. & $17,0 \mathrm{~kg} / \mathrm{mm} 2$ & Rigidez Dieléctrica & $8-12 \mathrm{kv} / \mathrm{mm}$ \\
\hline Módulo de elasticidad & $1,7.105 \mathrm{~kg} / \mathrm{mm} 2$ & Resistencia de impacto. & 20 Jouls \\
\hline Presión del test & 1,5 veces PS & Coef. Dilatación lineal & $15-10-6 \mathrm{~m} / \mathrm{m}^{\circ} \mathrm{C}$ \\
\hline
\end{tabular}
tructura de fibras cruzadas desordenadamente y gracias a la porosidad abierta, la lana mineral de vidrio permite que el aire quede ocluido en el interior de sus poros, ofreciendo un alto aislamiento acústico y escasa conductividad.

\section{TABLA III. Características de la fibra de vidrio}

Fibra de vidrio 
I.Diseño CAD

En esta etapa mediante software se diseña una es- tructura cilíndrica de 79,20 [mm] de diámetro y 93 [mm] de longitud, con paredes dobles de 1,5 y 2 [mm].
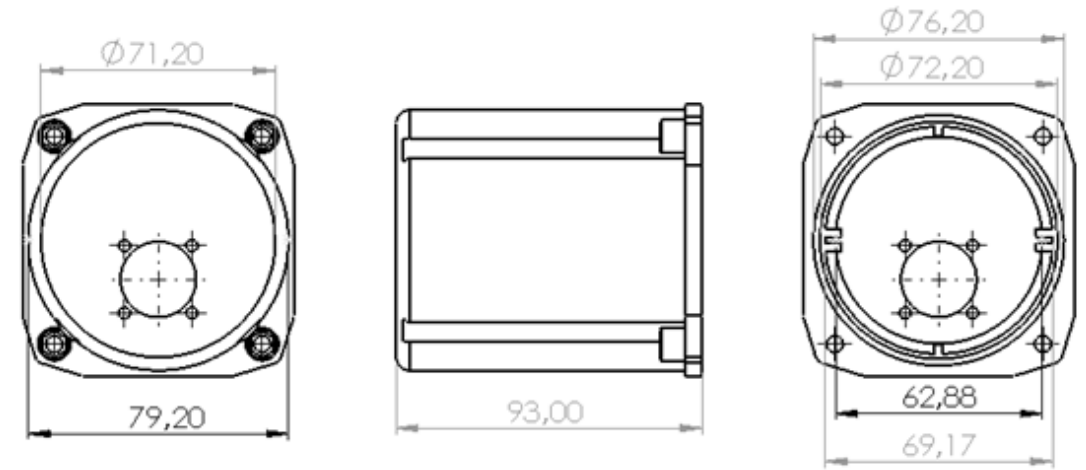

Fig. 7. Dimensiones físicas de la estructura del instrumento aeronáutico en ABS

\section{J.Simulación EMAG y CAE}

Para la validación del diseño se realiza la simulación del comportamiento de la estructura analizando por separado en campo eléctrico y magnético, esto mediante la asignación de condiciones de frontera como: ambiente, conductividad, dirección de propagación y magnitud
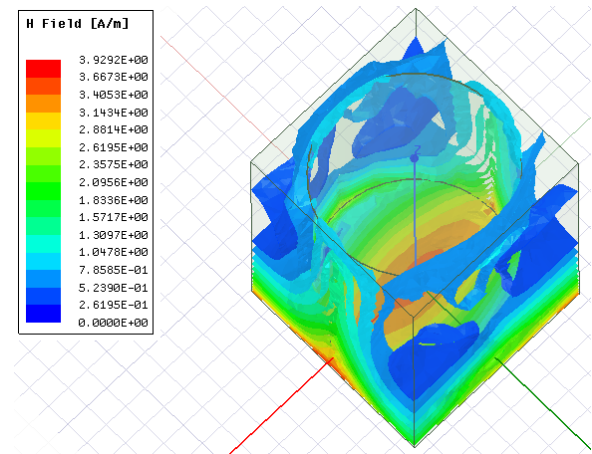

de oscilación [10]. Como se observa en la figura 8 (izq) el campo magnético se encuentra entre $0[\mathrm{~A} / \mathrm{m}]$ y 3,92 $[\mathrm{A} / \mathrm{m}]$ y en la figura 8 (der) el campo eléctrico se encuentra entre $0[\mathrm{~V} / \mathrm{m}]$ y $4,1183[\mathrm{~V} / \mathrm{m}]$, ambos campos no ingresan al interior de la jaula de Faraday implementada.

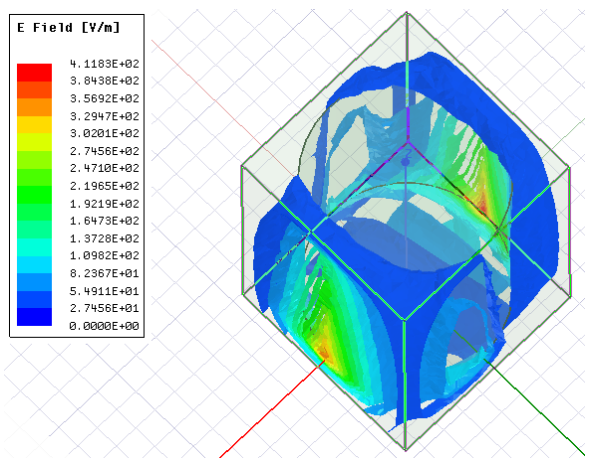

Fig. 8. Atenuación de la jaula de Faraday (izq) Campo magnético (der) Campo eléctrico

En la práctica la estructura no soporta grandes cargas, sin embargo, es necesario comprobar que la nueva estructura podrá proteger sus componentes internos. Para esto se toma como referencia teórica el módulo de Young (límite a la rotura) de los dos materiales (Aluminio y ABS) se determina que $y(\mathrm{Al} 7075)=500 \mathrm{Mpa}$

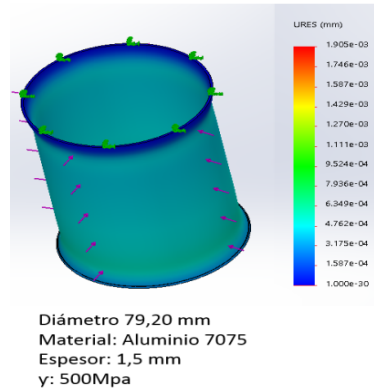

$>y(\mathrm{ABS})=40$, sin embargo estos son datos idealizados que pueden sufrir variaciones debido a otros factores como: geometría, dimensiones y refuerzos, lo cual es comprobable mediante un análisis estático asistido por computador.

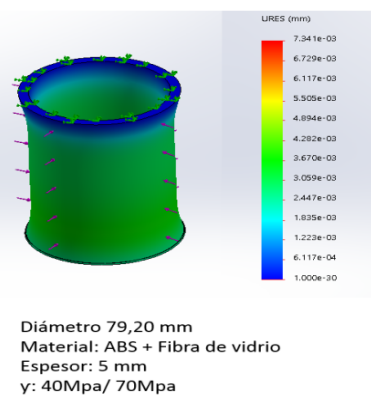

Fig. 9. Simulación estática mediante elementos finitos 
Al aplicar una fuerza similar de $500 \mathrm{~N}$ (límite de resistencia del aluminio) para ambos casos, se espera que por obvias razones el material de ABS falle prematuramente, sin embargo debido al mayor espesor de su construcción y la elasticidad del material se observa que la estructura de aluminio es más resistente en un $24 \%$, sin embargo el factor de seguridad es de 2,7 para el aluminio y 1,02 para el ABS, ubicando a este último por encima del valor deseado de uno determinando que es viable la utilización del material ABS en el diseño propuesto.

\section{K.Impresión 3D de la estructura}

Se utiliza la deposición fundida de material, en este caso filamento ABS de $1,75 \mathrm{~mm}$, que tiene $45 \mathrm{Mpa}$ de resistencia a la tracción y $200^{\circ} \mathrm{C}$ de temperatura de extrusión y luego de 36 horas se obtiene un sólido con un buen nivel de acabado y con soportes fácilmente removibles. Luego se agrega la malla y la fibra de vidrio en la cavidad en medio de las dos paredes.

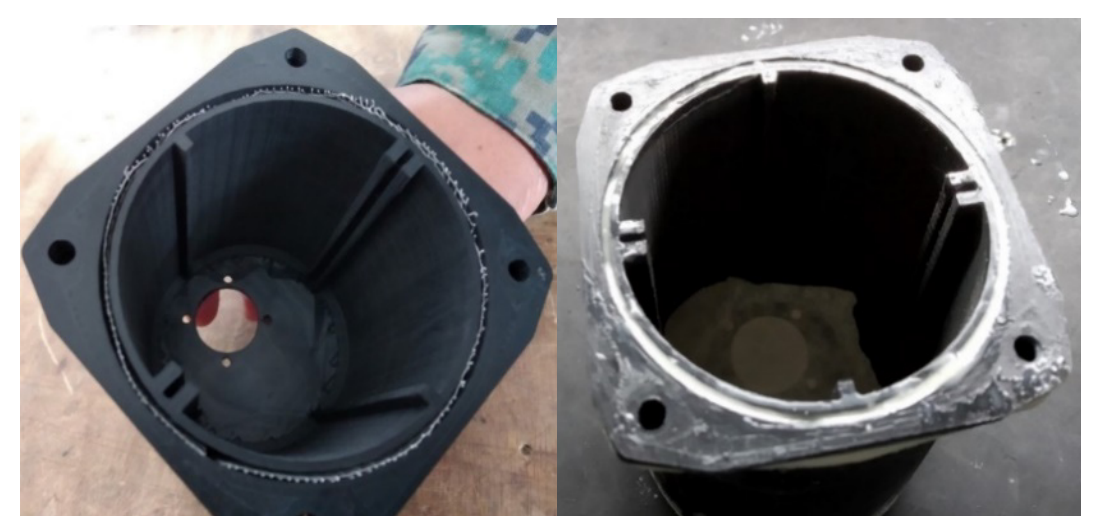

Fig. 10. Colocación de la malla y fibra de vidrio

Finalmente con el ensamblaje general de los componentes se dispone del nuevo instrumento.

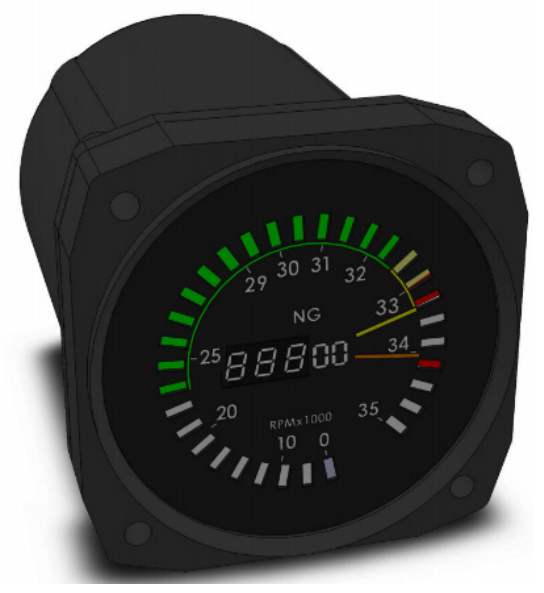

Fig. 11. Render del instrumento fabricado

\section{IV.RESULTADOS}

\section{A.Ensayos mecánicos}

Basados en las normas ASTM D638, ISO 527-2, ASTM D3039 e ISO 458 para polímeros obtenidos mediante manufactura aditiva se realizaron los ensayos mecánicos utilizando la máquina Metrotec Serie MTE50, y se obtuvieron los resultados presentados en la figura 12. 


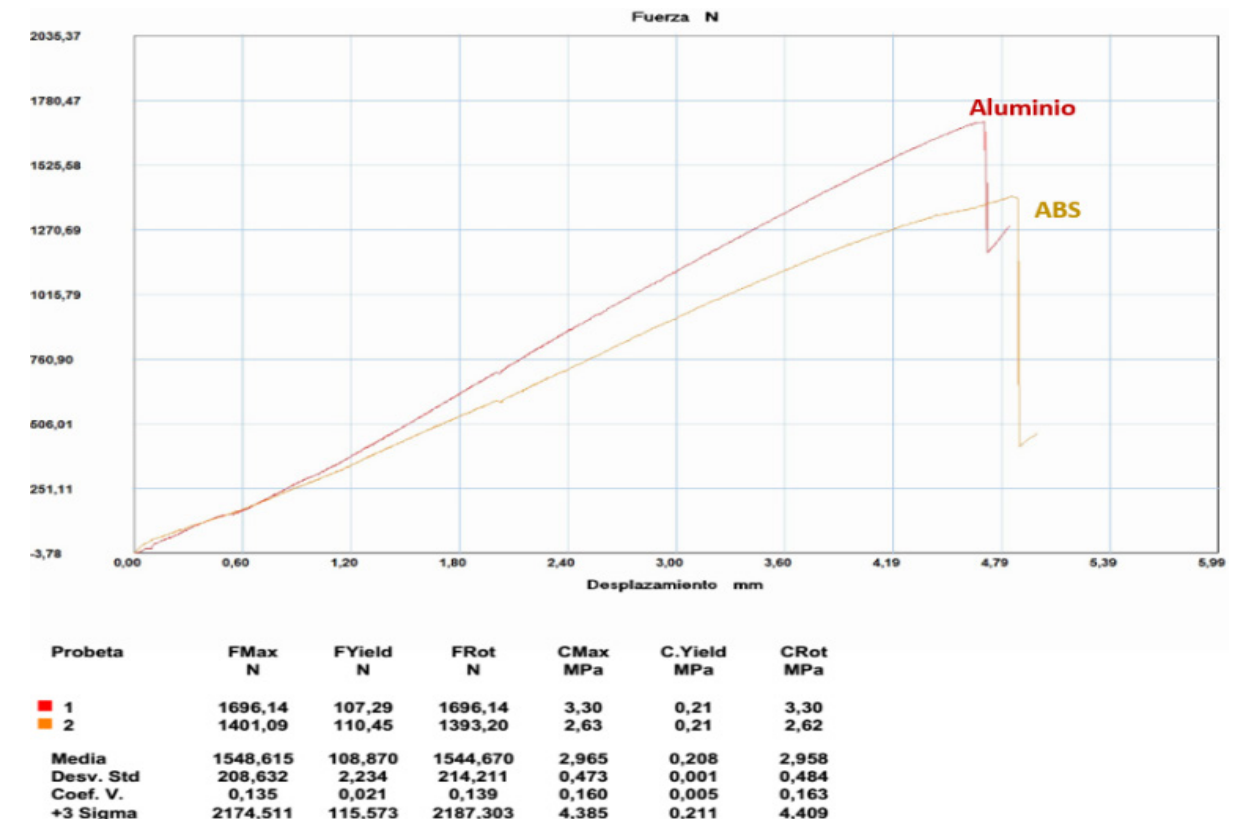

Fig. 12. Curvas esfuerzo deformación de las estructuras (Aluminio-ABS)

Las dos estructuras se comportaron de forma similar hasta los $251 \mathrm{~N}$, con valores superiores la estructura de ABS sufre una mayor deformación; el comportamiento resulta uniforme con una pendiente mayor para la estructura de aluminio (mayor resistencia), al final la estructura de ABS sufre la rotura ante 1401,09 N de fuerza, mientras que la estructura de aluminio lo hace ante $1696,14 \mathrm{~N}$, es decir la estructura en ABS tiene un $17,3 \%$ menor resistencia a la tracción que la estructura de aluminio embutido, sin embargo al no ser un factor crítico se puede determinar que la nueva estructura en plástico ABS puede ser aplicable en instrumentos de uso aeronáutico.

\section{B.Mediciones de campos electromagnéticos y ruido}

Empleando los helicópteros AS-332B y SA-330L (aeronaves que generan mayor nivel de ruido) y con los equipos de medición EMF Tester Electromagnetic Field AS1392 y Sound Level Meter GM1359 se tomaron muestras de los niveles de campos electromagnéticos (EMF) y ruido (SL) respectivamente, dentro y fuera de las estructuras de aluminio y ABS.

TABLA IV. Mediciones tomadas en el helicóptero AS-332B

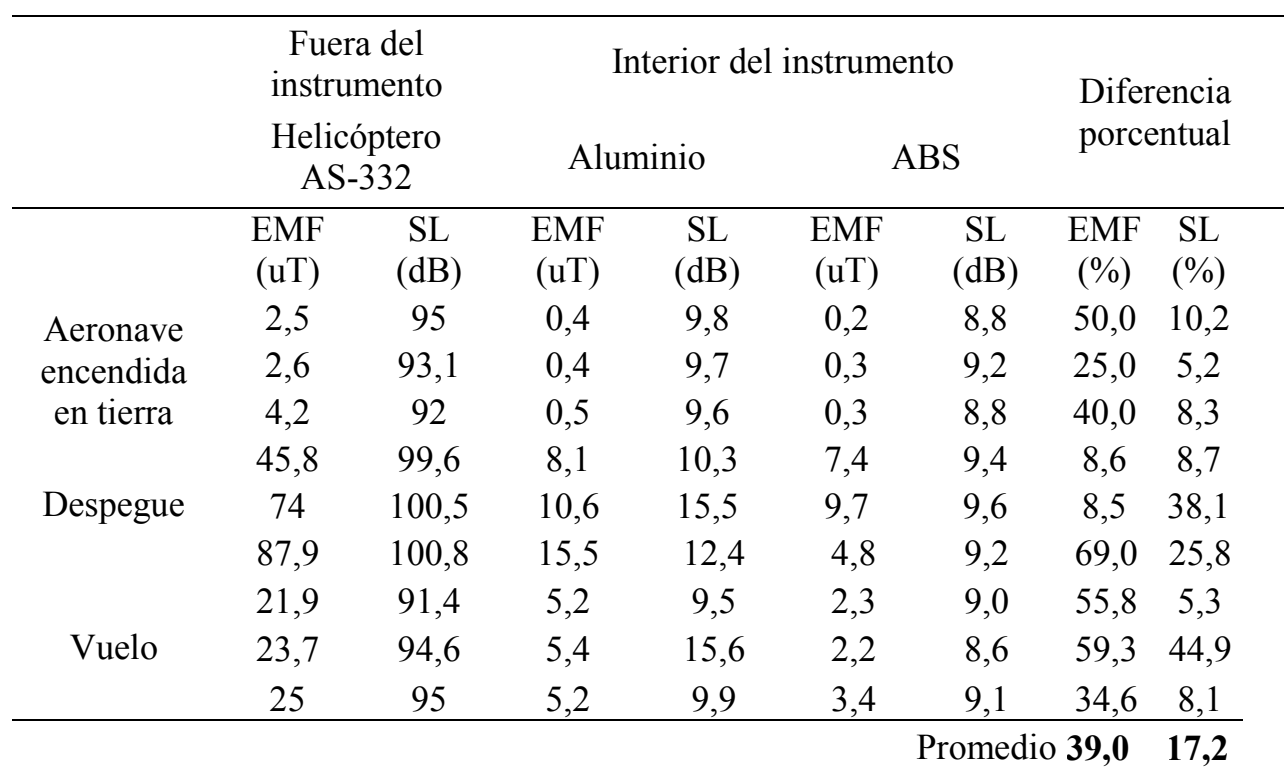


TABLA V. Mediciones tomadas en el helicóptero SA-330

\begin{tabular}{|c|c|c|c|c|c|c|c|c|}
\hline & \multirow{2}{*}{\multicolumn{2}{|c|}{$\begin{array}{c}\text { Fuera del } \\
\text { instrumento } \\
\text { Helicóptero } \\
\text { SA-330 }\end{array}$}} & \multicolumn{4}{|c|}{ Interior del instrumento } & & \\
\hline & & & \multicolumn{2}{|c|}{ Aluminio } & \multicolumn{2}{|c|}{ ABS } & \multicolumn{2}{|c|}{$\begin{array}{l}\text { Diferencia } \\
\text { porcentual }\end{array}$} \\
\hline & $\begin{array}{l}\text { EMF } \\
\text { (uT) }\end{array}$ & $\begin{array}{c}\text { SL } \\
(\mathrm{dB})\end{array}$ & $\begin{array}{l}\text { EMF } \\
\text { (uT) }\end{array}$ & $\begin{array}{c}\text { SL } \\
(\mathrm{dB})\end{array}$ & $\begin{array}{l}\text { EMF } \\
\text { (uT) }\end{array}$ & $\begin{array}{c}\text { SL } \\
(\mathrm{dB})\end{array}$ & $\begin{array}{c}\text { EMF } \\
(\%)\end{array}$ & $\begin{array}{l}\text { SL } \\
(\%)\end{array}$ \\
\hline \multirow{4}{*}{$\begin{array}{c}\text { Aeronave } \\
\text { encendida } \\
\text { en tierra }\end{array}$} & 3,4 & 100,2 & 0,4 & 8,9 & 0,2 & 8,0 & 50,0 & 10,1 \\
\hline & 3,7 & 100,3 & 0,4 & 8,8 & 0,2 & 8,3 & 50,0 & 5,7 \\
\hline & 4 & 100,5 & 0,5 & 8,8 & 0,3 & 8,1 & 40,0 & 8,0 \\
\hline & 101,5 & 105,6 & 7,2 & 9,3 & 6,7 & 8,6 & 6,9 & 7,5 \\
\hline \multirow[t]{3}{*}{ Despegue } & 102 & 105,9 & 10,0 & 14,1 & 8,9 & 8,8 & 11,0 & 37,6 \\
\hline & 105,6 & 106 & 14,1 & 11,2 & 4,5 & 8,4 & 68,1 & 25,0 \\
\hline & 30,1 & 99,1 & 4,7 & 8,7 & 2,3 & 8,2 & 51,1 & 5,7 \\
\hline \multirow[t]{2}{*}{ Vuelo } & 33,2 & 100,5 & 4,9 & 14,2 & 2,0 & 7,9 & 59,2 & 44,4 \\
\hline & 36 & 102,5 & 4,8 & 9,0 & 3,0 & 8,3 & 37,5 & 7,8 \\
\hline
\end{tabular}

Según la tabla $\mathrm{V}$, se determina que en el helicóptero AS-332B la estructura de ABS en comparación a la estructura de aluminio embutido tiene una mejor atenuación electromagnética en un 39\% y una mayor supresión de ruido en un 17,2\%. En la tabla 5, se observa un comportamiento similar en el helicóptero SA-330 con un 41,5\% más de atenuación electromagnética y mejora en un $16,9 \%$ en la supresión de ruido. Se obtiene un promedio porcentual con las características mejoradas de 40,25\% para la atenuación de campos electromagnéticos y $17,05 \%$ para la supresión de ruido.

\section{V.CONCLUSIONES}

1.La investigación del empleo de la impresión 3D en el área aeronáutica evidenció que es un proyecto viable y con altas expectativas para suplir la falta de repuestos de aviación.

2.El ensayo de tensión dio como resultado una menor resistencia mecánica en un $17,3 \%$ de la estructura de plástico ABS frente al aluminio.

3.La resistencia a la tracción no es un elemento crítico en las estructuras de instrumentos de uso aeronáutico.

4.Mediante el efecto de la jaula de Faraday fue posible la atenuación de los campos electromagnéticos con un margen superior de $40,25 \%$ frente a las estructuras de aluminio de los instrumentos aeronáuticos.

5.Empleando el principio de las paredes dobles, se logró una supresión de ruido superior en un $17,05 \%$ en comparación a las estructuras de aluminio de los instrumentos aeronáuticos.

\section{RECONOCIMIENTO}

El presente trabajo de investigación fue desarrollado gracias al apoyo del Centro de Mantenimiento Aéreo del Ejército Ecuatoriano, al cual extendemos nuestra gratitud por la facilidad para la utilización de las aeronaves de ala rotatoria.

\section{REFERENCIAS}

[1]R. Collinson, Introduction to Avionics Systems, Rochester, Kent, U.K.: Springer, 2013.

[2]G. M. P., Introducción a los procesos de manufactura, México: Mc Graw Hill, 2014.

[3]B. v. d. Berg, 3D Printing, Leiden : Springer, 2016. [4]Airbus, «Airbus Technical Magazine,» FAST Flight Airworthiness Support Technology, vol. único, $\mathrm{n}^{\circ}$ 55, p. 40, 2015.

[5]J. H. \&. L. Serway, Física para ciencias e Ingeniería, Filadelfia: Mc Graw Hill, 2002.

[6]M. Moser, Ingeniería Acústica, teoría y aplicaciones., Berlin: Springer, 2009.

[7]R. E. Olcina, «Radiación de energía electromagnética,» de Interferencias electromagnéticas en componentes electrónicos, Madrid, Dialnet, 1992, pp. 389-394.

[8]Y. Freedman, Física Universitaria, México: Pearson, 2009.

[9]B. P. N. Anderson, The Elecromagnetic Field, York, London: Springer, 1968.

[10]U. S. Dixit, Simulations for design and manufacturing, Singapore: Springer, 2018. 


\section{RESUMEN CURRICULAR}

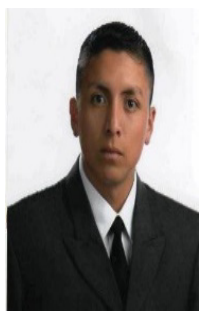

Héctor Lasluisa, ecuatoriano, en el 2008 obtuvo el título de Tlgo. Electrónico en la ESPE, en el 2011 obtuvo el título de Ing. Electrónico en la Escuela Politécnica Javeriana, en el 2017 se gradúa de Magíster en Manufactura y Diseño asistido por computador en la Universidad de las Fuerzas Armadas-ESPE. Actualmente se desempeña como investigador de Aplicaciones Militares en el CICTE-ESPE.

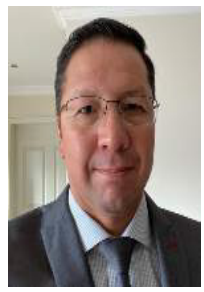

Aldo Capelo, ecuatoriano, en el 2008 obtuvo el título de Ing. Electrónico en la ESPE, el 2011 obtuvo el título de Magíster en Redes de Información y Conectividad en la ESPE, en el 2017 se gradúa de Magíster en Manufactura y Diseño asistido por computador en la Universidad de las Fuerzas Armadas-ESPE. Actualmente es asesor de proyectos tecnológicos en el CLT-ESPE 